\title{
Entanglement of Assistance is not a bipartite measure nor a tripartite monotone
}

\author{
Gilad Gour ${ }^{1, \text { 因 }}$ and Robert W. Spekkens ${ }^{2,3,0}$ \\ ${ }^{1}$ Department of Mathematics, University of California/San Diego, La Jolla, California 92093-0112 \\ ${ }^{2}$ Perimeter Institute for Theoretical Physics, Waterloo, Ontario N2L 2Y5, Canada \\ ${ }^{3}$ Institute for Quantum Computing, University of Waterloo, Ontario N2L 3G1, Canada
}

(Dated: Dec. 13, 2005)

\begin{abstract}
The entanglement of assistance quantifies the entanglement that can be generated between two parties, Alice and Bob, given assistance from a third party, Charlie, when the three share a tripartite state and where the assistance consists of Charlie initially performing a measurement on his share and communicating the result to Alice and Bob through a one-way classical channel. We argue that if this quantity is to be considered an operational measure of entanglement, then it must be understood to be a tripartite rather than a bipartite measure. We compare it with a distinct tripartite measure that quantifies the entanglement that can be generated between Alice and Bob when they are allowed to make use of a two-way classical channel with Charlie. We show that the latter quantity, which we call the entanglement of collaboration, can be greater than the entanglement of assistance. This demonstrates that the entanglement of assistance (considered as a tripartite measure of entanglement), and its multipartite generalizations such as the localizable entanglement, are not entanglement monotones, thereby undermining their operational significance.
\end{abstract}

PACS numbers: 03.67.Mn, 03.67.Hk, 03.65.Ud

The first significant progress to be made on the problem of quantifying entanglement came from the recognition that an entangled state is a resource for distributed quantum information processing (QIP) using local operations and classical communication (LOCC) 1]. This led to the idea that one could define many operational measures of the entanglement of a state as follows. For every QIP task imaginable, quantify the success with which one could achieve this task given LOCC and one or more copies of the entangled state in question. In order for the degree of success to be uniquely defined, one must perform an optimization over all protocols for achieving the task subject to the LOCC restriction. Clearly then, the degree of success must be nonincreasing under LOCC, so that if we take a measure of entanglement to be simply a measure of the success with which one can achieve a distributed QIP task under LOCC, then any such measure must be a monotone under LOCC [2, 3, 4].

The introduction of the notion of an entanglement monotone has provided a framework in which to make sense of the myriad different measures of entanglement that have been proposed in the past few years. Because the requirement of monotonicity is relatively weak it has tended to lead to an inclusive attitude towards proposed measures of entanglement. It is remarkable therefore that this very weak requirement fails to be met by an entanglement measure that has been widely discussed, namely, the entanglement of assistance (EoA) [5, 6], as we will show. Because the EoA is a special case of the localizable entanglement (LE) [], another measure that has recently received significant attention, the latter is also

\footnotetext{
*Electronic address: ggour@math.ucsd.edu

${ }^{\dagger}$ Electronic address: rspekkens@perimeterinstitute.ca
}

not an entanglement monotone in general. This result calls into question the significance of the EoA and LE as operational measures of entanglement, that is, as measures of a resource for distributed QIP.

The original definition of the EoA is as follows. Suppose that Alice and Bob possess a bipartite system in the mixed state $\rho_{A B}$ and Charlie holds a purification of this state, which is to say that the tripartite system held by Alice, Bob and Charlie is in a pure state $|\Psi\rangle_{\Lambda B C}$ such that $\rho_{A B}=\operatorname{Tr}_{C}|\Psi\rangle_{A B C}\langle\Psi|$. The EoA of Ref. [5] quantifies the maximum average amount of pure-state entanglement (quantified by the entropy of entanglement $E_{\text {Ent }}$ ) that Alice and Bob can extract from $\rho_{A B}$ by Charlie performing a measurement on his system and reporting the outcome to Alice and Bob. It follows from the HughstonJozsa-Wootters (HJW) theorem [8] that

$$
E_{\mathrm{Ast}}\left(\rho_{A B}\right)=\max _{\left\{p_{i},\left|\psi_{i}\right\rangle_{A B}\right\}} \sum_{i} p_{i} E_{\mathrm{Ent}}\left(\left|\psi_{i}\right\rangle_{A B}\right)
$$

where the entropy of entanglement of $\left|\psi_{i}\right\rangle_{A B}$ is the von Neumann entropy of the reduced density operator on $A$; that is, $E_{\text {Ent }}\left(\left|\psi_{i}\right\rangle_{A B}\right)=S\left(\rho_{A, i}\right)$ with $\rho_{A, i} \equiv$ $\operatorname{Tr}_{B}\left(\left|\psi_{i}\right\rangle_{A B}\left\langle\psi_{i}\right|\right)$ and $S(\rho) \equiv-\operatorname{Tr}(\rho \log \rho)$. The maximization in (1) is over all convex decompositions of $\rho_{A B}$ into pure states; that is, all pure state ensembles $\left\{p_{i},\left|\psi_{i}\right\rangle_{A B}\right\}$ such that $\rho_{A B}=\sum_{i} p_{i}\left|\psi_{i}\right\rangle_{A B}\left\langle\psi_{i}\right|$.

Written in this form, it might seem that the EoA is a measure of entanglement for bipartite states, that is, a measure of the degree of success that can be achieved for some distributed QIP task given $\rho_{A B}$ and LOCC. Indeed, in the article that introduced the EoA, it is described as "a new quantification of entanglement of a general (mixed) quantum state for a bipartite system" [5]. However, it can happen that Alice and Bob's bipartite system is described by the mixed state $\rho_{A B}$ even though Charlie's system does not purify this state. For instance, 
the tripartite system might be in a mixed state $\rho_{A B C}$ such that $\rho_{A B}=\operatorname{Tr}_{C} \rho_{A B C}$. So we see that the definition privded by Eq. (11) does not quantify the usefulness of the bipartite state $\rho_{A B}$ but rather that of the tripartite state $|\Psi\rangle_{A B C}$. Thus, the EoA is clearly an operational measure of entanglement for tripartite states.

Suppose, however, that one tried to defend the notion that the EoA is a bipartite measure of entanglement by simply taking Eq. (1) as its definition, and dispensing with any reference to a tripartite state shared with Charlie. One can do so, but it is then trivial to show that such a measure is not a monotone. It suffices to note that one can always increase the EoA by simply discarding information. For instance, a product state for two qubits, which has $E_{\text {Ast }}=0$, may be transformed into the completely mixed state, which has $E_{\mathrm{Ast}}=1$ (this follows from the fact that such a state can be decomposed into an equal mixture of the four Bell states), by simply adding noise. The problem is that the EoA is a concave rather than a convex function [23].

It might appear that this puts the nails in the coffin of the notion that the EoA is a bipartite measure of entanglement, but one can in fact go much further. By adopting a very loose policy with regard to what should be called a measure of entanglement, one might consider dropping the requirement of monotonicity under LOCC operations and maintaining that the EoA is a bipartite measure of entanglement in a broader sense. However, for a measure to be considered an operational measure of entanglement in any sense whatsoever it must quantify the ability of the state to act as a nonlocal resource for some set of restricted operations. In other words, there must be some set of operations, more restrictive than LOCC, such that it is nonincreasing under these. For instance, one might think that by forbidding the discarding of information, to which we appealed in the example above, the EoA might become nonincreasing under the remaining operations. As it turns out, this is not the case. One can find examples of states for which the EoA increases even under LOCC with no discarding of information. What if we imagine adding the restriction of no classical communication? This doesn't work either: the EoA is still not a monotone. What about adding the restriction of no generalized measurements, that is, forbidding the use of an ancilla? One finds that the EoA is still not a monotone. In fact, the central example of our paper, the state (2), shows that even if one forbids all operations save one, namely, a single two-outcome projective measurement implemented by a single party, the EoA is still not found to be a monotone because it can be increased by this operation. Thus, we conclude that there is no sense in which the EoA can be considered to be an operational measure of entanglement for bipartite systems.

For the rest of this article we consider the EoA to be a tripartite measure of entanglement and we address the question of whether or not it is a tripartite monotone, that is, whether or not it is nonincreasing under
LOCC operations among all three parties. To establish the failure of monotonicity of the EoA as a tripartite measure of entanglement, we will compare it to another tripartite measure which we shall call the entanglement of collaboration (EoC). It is defined as the maximum average amount of pure-state entanglement (quantified by the entropy of entanglement $E_{\text {Ent }}$ ) that Alice and Bob can obtain starting from a tripartite state $|\Psi\rangle_{A B C}$ with reduction $\rho_{A B}$, after arbitrary tripartite LOCC operations between Alice, Bob and Charlie. The EoC differs from the EoA because it allows Alice and Bob to assist Charlie in assisting them (hence "collaboration").

The EoA and the EoC are similarly motivated - they both seek to quantify how much better Alice and Bob can make use of their entanglement given some kind of help from Charlie. It is likely that the EoA has been studied preferentially over EoC simply because of the greater difficulty involved in computing the $\mathrm{EoC}$ - the extra level of assistance that arises in its definition requires one to consider an arbitrary number of rounds of communication with optimizations at every round. However, what has perhaps gone unnoticed in opting to solve the simpler of the two problems is that if there exist states for which the EoC is greater than the EoA, then the EoA is not an entanglement monotone. This is so because in this case it would be possible to increase the EoA by an LOCC operation - namely one wherein Alice and Bob communicate with Charlie (either once or in the course of many rounds of communication). We shall presently demonstrate an example of a state for which this is the case.

The example makes use of a tripartite system of dimensionality $8 \times 4 \times 2$. The state is:

$$
\begin{aligned}
|\Phi\rangle_{A B C}= & \frac{1}{4}[(|00\rangle+|11\rangle+|22\rangle+|33\rangle)|0\rangle \\
& +(i|00\rangle+|11\rangle-i|22\rangle-|33\rangle)|1\rangle \\
& +(|40\rangle+|51\rangle+|62\rangle+|73\rangle)|+i\rangle \\
& +(i|40\rangle+|51\rangle+i|62\rangle+|73\rangle)|-i\rangle]
\end{aligned}
$$

where $| \pm i\rangle \equiv(|0\rangle \pm i|1\rangle) / \sqrt{2}$.

An optimal collaborative scheme proceeds as follows. Alice first implements a measurement with Kraus operators $|0\rangle\langle 0|+| 1\rangle\langle 1|+| 2\rangle\langle 2|+| 3\rangle\langle 3|$ and $|4\rangle\langle 4|+| 5\rangle\langle 5|+$ $|6\rangle\langle 6|+| 7\rangle\langle 7|$ and sends the outcome to Charlie. If the first outcome has occurred, Charlie measures the $\{|0\rangle,|1\rangle\}$ basis, otherwise he measures the $\{|+i\rangle,|-i\rangle\}$ basis. In each case, regardless of the outcome, he is sure to leave Alice and Bob with a state that is maximally entangled in the $8 \times 4$ Hilbert space. The scheme is optimal because it always achieves a maximally entangled state. When measured by the entropy of entanglement, the $\mathrm{EoC}$ in this case is 2 e-bits.

On the other hand, if no communication from Alice and Bob to Charlie is allowed, then he can not create a state that is maximally entangled between Alice and Bob 
in the $8 \times 4$ Hilbert space so that the EoA is strictly less than 2 e-bits.

The proof is as follows. First note that the state $\Psi$ above can be written as follows:

$$
|\Phi\rangle_{A B C}=\left|u_{0}\right\rangle_{A B}|0\rangle_{C}+\left|u_{1}\right\rangle_{A B}|1\rangle_{C}
$$

where the $8 \times 4$ sub-normalized states $\left|u_{0}\right\rangle_{A B}$ and $\left|u_{1}\right\rangle_{A B}$ are given by their Schmidt decomposition forms:

$$
\begin{aligned}
&\left|u_{0}\right\rangle_{A B} \equiv \sum_{k=0}^{3}\left|c_{0, k}\right\rangle|k\rangle \equiv \frac{1}{4}[(|0\rangle+z|4\rangle)|0\rangle \\
&+(|1\rangle+\sqrt{2}|5\rangle)|1\rangle+(|2\rangle+z|6\rangle)|2\rangle+(|3\rangle+\sqrt{2}|7\rangle)|3\rangle] \\
&\left|u_{1}\right\rangle_{A B} \equiv \sum_{k=0}^{3}\left|c_{1, k}\right\rangle|k\rangle \equiv \frac{1}{4}[(i|0\rangle+z|4\rangle)|0\rangle+|1\rangle|1\rangle \\
&+(-i|2\rangle+z|6\rangle)|2\rangle-|3\rangle|3\rangle]
\end{aligned}
$$

where $z \equiv(1+i) / \sqrt{2}$. The reduced density matrix $\rho_{A B}=$ $\operatorname{Tr}_{C}|\Phi\rangle_{A B C}\langle\Phi|$ is therefore given by:

$$
\rho_{A B}=\left|u_{0}\right\rangle_{A B}\left\langle u_{0}|+| u_{1}\right\rangle_{A B}\left\langle u_{1}\right|
$$

We now show that Charlie can not always prepare a state that is maximally entangled in $8 \times 4$. In fact, we will show something stronger: Charlie can not even prepare an $8 \times 4$ state of maximal entanglement with some probability less then one. Given the HJW theorem, it is enough to show that no convex decomposition of $\rho_{A B}$ contains any such states. To see this, note that any state in some decomposition of $\rho_{A B}$ is proportional to a linear combination of $\left|u_{0}\right\rangle_{A B}$ and $\left|u_{1}\right\rangle_{A B}$. Therefore, it is enough to show that any normalized state of the form $x\left|u_{0}\right\rangle_{A B}+y\left|u_{1}\right\rangle_{A B}$, with $x$ and $y$ complex, is not an $8 \times 4$ maximally entangled state.

If $x\left|u_{0}\right\rangle_{A B}+y\left|u_{1}\right\rangle_{A B}$ is maximally entangled then all of its Schmidt coefficients must be of equal magnitude (and non-zero). The magnitudes of these coefficients are:

$$
\begin{aligned}
& \lambda_{1}=\frac{1}{16}\left(|x+i y|^{2}+\frac{1}{2}|x+y|^{2}\right) \\
& \lambda_{2}=\frac{1}{16}\left(|x+y|^{2}+2|x|^{2}\right) \\
& \lambda_{3}=\frac{1}{16}\left(|x-i y|^{2}+\frac{1}{2}|x+y|^{2}\right) \\
& \lambda_{4}=\frac{1}{16}\left(|x-y|^{2}+2|x|^{2}\right) .
\end{aligned}
$$

Thus, $\lambda_{1}=\lambda_{2}=\lambda_{3}=\lambda_{4}$ iff $x=y=0$. Consequently, any normalized state $x\left|u_{0}\right\rangle_{A B}+y\left|u_{1}\right\rangle_{A B}$ cannot be maximally entangled. This concludes the proof.

Entanglement of assistance for more than one copy - We define the $n$-copy EoA as follows:

$$
E_{\text {Ast }}^{(n)}\left(|\Psi\rangle_{A B C}\right) \equiv \frac{1}{n} E_{\text {Ast }}\left(\rho_{A B}^{\otimes n}\right)
$$

where $|\Psi\rangle_{A B C}$ is a tripartite pure state and $\rho_{A B} \equiv$ $\operatorname{Tr}_{C}|\Psi\rangle_{A B C}\langle\Psi|$.

It follows from the results in 10, 11], that in the asymptotic limit of infinitely many copies of a tripartite (multipartite) pure state, the EoA (LE) is indeed an entanglement monotone. That is, the function $E_{\text {Ast }}^{(\infty)}\left(|\Psi\rangle_{A B C}\right) \equiv$ $\lim _{n \rightarrow \infty} E_{\text {Ast }}^{(n)}\left(|\Psi\rangle_{A B C}\right)$ does not increase under tripartite LOCC 24]. However, for any finite integer $n<\infty$, the function $E_{\mathrm{Ast}}^{(n)}$ is not an entanglement monotone. This follows from the fact that for the state $|\Phi\rangle$ given in Eq. (2), the EoC is 2 ebits (having additional copies does not change this fact), while $E_{\text {Ast }}^{(n)}(|\Phi\rangle)<2$, as we now show.

From Eq. (5) and the HJW theorem it follows that any $8^{n} \times 4^{n}$-dimensional state in some decomposition of $\rho_{A B}^{\otimes n}$ can be written as a linear combination of the $2^{n}$ states $\left|u_{i_{1}} u_{i_{2}} \cdots u_{i_{n}}\right\rangle$, where $i_{m} \in\{0,1\}(m=1,2, \ldots, n)$. Thus, we want to show that any linear combination of $\left|u_{i_{1}} u_{i_{2}} \cdots u_{i_{n}}\right\rangle$ is not proportional to a maximally entangled state in the $8^{n} \times 4^{n}$-dimensional Hilbert space. We will prove it by induction.

For $n=1$ we have proved it above. Let us now assume that any linear combination of $\left|u_{i_{1}} u_{i_{2}} \cdots u_{i_{n}}\right\rangle$ is not proportional to a maximally entangled state and let $|\chi\rangle$ be a linear combination of the $2^{n+1}$ states $\left|u_{i_{1}} u_{i_{2}} \cdots u_{i_{n}} u_{i_{n+1}}\right\rangle$. Thus, $|\chi\rangle$ can be written as $\left|\chi_{0}\right\rangle\left|u_{0}\right\rangle+\left|\chi_{1}\right\rangle\left|u_{1}\right\rangle$, where $\left|\chi_{0}\right\rangle$ and $\left|\chi_{1}\right\rangle$ are some linear combinations of the states $\left|u_{i_{1}} u_{i_{2}} \cdots u_{i_{n}}\right\rangle$.

Let $b \in\{0,1\}$. From Eq. (4) and the property that $\left\langle c_{b, k} \mid c_{b^{\prime}, k^{\prime}}\right\rangle=0$ if $k \neq k^{\prime}$, one can deduce that $\left|\chi_{b}\right\rangle$ can be written in the form $\left|\chi_{b}\right\rangle=$ $\sum_{s=0}^{4^{n}-1}\left|\alpha_{b, s}\right\rangle|s\rangle$, where the subnormalized states, $\left|\alpha_{b, s}\right\rangle$, satisfy $\left\langle\alpha_{b, s} \mid \alpha_{b^{\prime}, s^{\prime}}\right\rangle=0$ if $s \neq s^{\prime}$. Thus, it follows that the Schmidt decomposition of $|\chi\rangle$ has the form $|\chi\rangle=$ $\sum_{s=0}^{4^{n}-1} \sum_{k=0}^{3}\left(\sum_{b=0}^{1}\left|\alpha_{b, s}\right\rangle\left|c_{b, k}\right\rangle\right)|s\rangle|k\rangle$. Defining $\mu_{b, s} \equiv$ $\left\langle\alpha_{b, s} \mid \alpha_{b, s}\right\rangle$ and $\eta_{s} \equiv\left\langle\alpha_{0, s} \mid \alpha_{1, s}\right\rangle$, the Schmidt coefficients of $|\chi\rangle$ may be expressed as $\lambda_{s, k}=\sum_{b=0}^{1} \mu_{b, s}\left\langle c_{b, k} \mid c_{b, k}\right\rangle+$ $2 \operatorname{Re}\left[\eta_{s}\left\langle c_{0, k} \mid c_{1, k}\right\rangle\right]$. From Eq. (4), one can deduce that

$$
\begin{aligned}
& \lambda_{s, 0}=\frac{1}{8} \mu_{0, s}+\frac{1}{8} \mu_{1, s}+\frac{1}{8} \operatorname{Re}\left[(1+i) \eta_{s}\right] \\
& \lambda_{s, 1}=\frac{3}{16} \mu_{0, s}+\frac{1}{16} \mu_{1, s}+\frac{1}{8} \operatorname{Re}\left[\eta_{s}\right] \\
& \lambda_{s, 2}=\frac{1}{8} \mu_{0, s}+\frac{1}{8} \mu_{1, s}+\frac{1}{8} \operatorname{Re}\left[(1-i) \eta_{s}\right] \\
& \lambda_{s, 3}=\frac{3}{16} \mu_{0, s}+\frac{1}{16} \mu_{1, s}-\frac{1}{8} \operatorname{Re}\left[\eta_{s}\right] .
\end{aligned}
$$

For $|\chi\rangle$ to be proportional to a maximally entangled state, the $\lambda_{s, k}$ must be equal, which implies that $\eta_{s}=0$, $\mu_{0, s}=\mu_{1, s}$ for all $s$ and $\mu_{b, s}=\mu_{b, s^{\prime}}$ for all $s$ and $s^{\prime}$. But by the assumption of our inductive proof, $\left|\chi_{b}\right\rangle$ is not maximally entangled and therefore $\mu_{b, s} \neq \mu_{b, s^{\prime}}$ for some $s$ and $s^{\prime}$ and so $|\chi\rangle$ is not maximally entangled either. This concludes the inductive proof.

So we see that the n-copy EoA, $E_{\mathrm{Ast}}^{(n)}$, is not a monotone whereas the asymptotic version of this quantity, $E_{\text {Ast }}^{(\infty)} \equiv \lim _{n \rightarrow \infty} E_{\text {Ast }}^{(n)}$, is. Thus, we have found that 
regularization yields monotonicity. It is interesting to note that this also occurs for the classical information deficit, a measure of the classical correlations of a quantum state 12, 13]. Specifically, the single-copy version of the classical deficit is not a monotone, as shown in Ref. 13], while the results of Ref. [14] demonstrate that the regularized version of the classical deficit is equal to the one-way distillable common randomness [15, 16], which is a monotone [25].

Generalizations of the entanglement of assistance - Although the EoA was defined in terms of the entropy of entanglement in Eq. (11), one could equally well have chosen any measure of entanglement for bipartite pure states without compromising the appropriateness of the name "entanglement of assistance". We therefore follow 7, 18, 19, 20] in generalizing the definition of EoA to constitute a family of measures, with each one being parasitic on (i.e. defined in terms of) a different bipartite measure of entanglement. The latter is required to be an entanglement monotone with respect to LOCC operations on the bipartite system. We shall call it the root entanglement measure and denote it by $E_{\mathrm{Rt}}$.

Thus, given a quantum state of a tripartite system, the EoA is defined as the maximum average of the root entanglement measure that Alice and Bob can share after Charlie has performed a local operation and communicated the result to Alice and Bob. Note that this manner of defining the EoA extends readily to mixed tripartite states as long as $E_{\mathrm{Rt}}$ is a measure of entanglement that is defined for mixed bipartite states. Similarly we can define the EoC for any given root entanglement measure.

The example of Eq. (2) demonstrates that the EoC is greater than the EoA as long as the root entanglement measure can distinguish maximally entangled states from nonmaximally entangled states, which is to say that it assigns different values to these states. For pure states, this is a property that is satisfied, for instance, by the von Neumann entropy but not, for instance, by the Schmidt number. Thus our example implies the following general result: the EoA fails to be an entanglement monotone for any root entanglement measure that can distinguish maximally entangled states from nonmaximally entangled states.

Our results are also significant for a quantity known as the localizable entanglement (LE). Given a state of a multipartite system, the LE is the maximum average of the root entanglement measure that Alice and Bob can share after LOCC operations among the other parties [26]. The generalization of LE wherein one optimizes over LOCC operations between all the parties, we call the collaboratively localizable entanglement (CLE). Clearly, the EoA (EoC) is simply the LE (CLE) in the case where there is only one additional party. As such, our tripartite example demonstrates that CLE may be greater than the LE and consequently we conclude that the LE is not an entanglement monotone either.

An example of smaller dimensionality using a mixed tripartite state - Here we give an example of a mixed state on a tripartite system of dimensionality $4 \times 2 \times 2$ for which the EoC is greater than the EoA. The state is an equal mixture of the four pure states

$$
\begin{aligned}
\left|\phi_{1}\right\rangle_{A B}|0\rangle_{C} & \equiv \frac{1}{\sqrt{2}}\left(|0\rangle_{A}|1\rangle_{B}+|1\rangle_{A}|0\rangle_{B}\right)|0\rangle_{C}, \\
\left|\phi_{2}\right\rangle_{A B}|1\rangle_{C} & \equiv \frac{1}{\sqrt{2}}\left(|0\rangle_{A}|0\rangle_{B}+|1\rangle_{A}|1\rangle_{B}\right)|1\rangle_{C} . \\
\left|\phi_{3}\right\rangle_{A B}|+\rangle_{C} & \equiv \frac{1}{\sqrt{2}}\left(|2\rangle_{A}|1\rangle_{B}+|3\rangle_{A}|0\rangle_{B}\right)|+\rangle_{C}, \\
\left|\phi_{4}\right\rangle_{A B}|-\rangle_{C} & \equiv \frac{1}{\sqrt{2}}\left(|2\rangle_{A}|0\rangle_{B}+|3\rangle_{A}|1\rangle_{B}\right)|-\rangle_{C},
\end{aligned}
$$

In the optimal collaborative scheme, Alice implements a projective measurement with Kraus operators $|0\rangle\langle 0|+$ $|1\rangle\langle 1|$ and $|2\rangle\langle 2|+| 3\rangle\langle 3|$ and communicates her outcome to Charlie, who then knows whether to measure in the $\{|0\rangle,|1\rangle\}$ or $\{|+\rangle,|-\rangle\}$ bases in order to be sure to collapse $A B$ to one of the $\left|\phi_{i}\right\rangle$. The scheme is optimal because these are maximally entangled in the $4 \times 2$ space. A scheme consisting purely of assistance by Charlie cannot do as well because for any generalized measurement that Charlie performs, he leaves $A B$ in a mixture of the $\left|\phi_{i}\right\rangle$ with at least three of these states receiving non-zero weight, and it can be shown that no such mixture is maximally entangled in $4 \times 2$.

Discussion - In the presence of a noisy environment, creating and distributing entanglement on a multi-user quantum network is a challenging problem. The question of precisely how much entanglement can be created between a chosen pair of parties starting from an arbitrary multi-party state is therefore an important one. In the introduction it was argued that any measure of entanglement that sought to quantify the degree of success of some distributed QIP task must be a monotone under LOCC, and entanglement creation on a network certainly qualifies as such a task. This leads us to the following puzzle. The EoA and LE certainly seem to have some operational significance for the problem of entanglement creation, and yet we have just shown that they fail to be monotones. How can this be?

The puzzle is resolved by noting that the EoA and LE do quantify the usefulness of a quantum state for certain tasks, but these are not the sorts of tasks that one typically considers, namely, distributed QIP tasks given LOCC. Rather, they quantify the usefulness of a quantum state for distributed QIP tasks given local operations and restricted classical communication, where the restriction is that there is no classical communication allowed from Alice and Bob to the other parties. The EoA and LE are strictly non-increasing under these restricted operations. We may say that they are monotones, but only with respect to this restricted version of LOCC.

It follows that EoA and LE are operationally significant only if one is faced with a network that places the specified restrictions on classical communications. However, such a restriction is unlikely to arise naturally in practical quantum information networks and conse- 
quently for such applications it is the EoC and CLE rather than the EoA and LE that are the quantities that are of the most interest.

In addition to its obvious applications in the field of quantum communication and the study of multipartite entanglement, the concept of LE has proven to be fruitful in the field of condensed matter physics, in particular for understanding the complex physics of strongly correlated states (for example, see references in [7]). Our results are therefore likely to have applications in this area. For instance, the entanglement length, which characterizes the typical length up to which bipartite entanglement can be localized in the system, can be, in general, greater when defined in terms of the CLE rather then the LE.

There was a significant caveat in the statement of our main result: we have only shown that EoA and LE fail to be entanglement monotones (the usual kind, with respect to unrestricted LOCC) when they are defined in terms of a root entanglement measure that can distinguish maximally entangled states from nonmaximally entangled states. This leaves open the possibility that the EoA and LE can be entanglement monotones for choices of the root measure that are not of this sort. Elsewhere we show that this possibility is indeed realized in the case where the root measure is the G-concurrence, a generalization of the concurrence 21] to pairs of systems of dimensionality greater than 2 [20, 22]. Also, if Alice and Bob's systems are of smaller dimensionality than considered here, then there will likely be more root entanglement measures for which the EoA is found to be a monotone. Indeed, for $2 \times 2 \times 2$ pure states if the root measure is the concurrence (which does distinguish maximally from nonmaximally entangled states), then the resulting EoA can be proven to be a monotone [19]. Determining in which Hilbert spaces and for which root entanglement measures the EoA is a monotone will help to identify those distributed QIP tasks for which having a collaboration with Charlie provides no advantage over merely having his assistance.

Acknowledgments:- The authors gratefully acknowledge Michal Horodecki, Debbie Leung, Michael Nielsen, Jonathan Oppenheim and Martin Plenio for helpful comments. G.G. acknowledge support by the National Science Foundation (NSF) under Grant No. ECS-0202087.
[1] C. H. Bennett, H. J. Bernstein, S. Popescu, B. Schumacher, Phys. Rev. A 53, 2046 (1996).

[2] S. Popescu and D. Rohrlich, Phys. Rev. A 56, R3319 (1997).

[3] V. Vedral and M. B. Plenio, Phys. Rev. A 57, 1619 (1998).

[4] G. Vidal, J. Mod. Opt. 47, 355 (2000).

[5] D. P. DiVincenzo et al, "The entanglement of assistance", in Lecture Notes in Computer Science 1509 (SpringerVerlag, Berlin, 1999), pp. 247-257.

[6] O. Cohen, Phys. Rev. Lett. 80, 2493 (1998).

[7] M. Popp, F. Verstraete, M. A. Martin-Delgado and J. I. Cirac, Phys. Rev. A 71, 042306 (2005).

[8] L. P. Hughston, R. Jozsa and W. K. Wootters, Phys. Lett. A 183, 14 (1993).

[9] M. B. Plenio, Phys. Rev. Lett. 95, 090503 (2005).

[10] J. A. Smolin, F. Verstraete, and A. Winter, Phys. Rev. A, 72, 052317 (2005).

[11] M. Horodecki, J. Oppenheim and A. Winter, Nature 436, 673 (2005).

[12] M. Horodecki, K. Horodecki, P. Horodecki, R. Horodecki, J. Oppenheim, A. Sen(De) and U. Sen, Phys. Rev. Lett. 90, 100402 (2003).

[13] B. Synak and M. Horodecki, J. Phys. A 37, 11465 (2004).

[14] I. Devetak, quant-ph/0406234

[15] L. Henderson and V. Vedral, J. Phys. A 34, 6899 (2001).
[16] I. Devetak and A. Winter, IEEE Transactions on Information Theory 50, 3183 (2004).

[17] C. H. Bennett, D. P. DiVincenzo, J. A. Smolin and W. K. Wootters, Phys.Rev. A 54, 3824 (1996).

[18] T. Laustsen, F. Verstraete, and S. J. van Enk, Quant. Inf. and Comp. 3, 64 (2003).

[19] G. Gour, D. A. Meyer and B. C. Sanders, Phys. Rev. A 72, 042329 (2005).

[20] G. Gour, Phys. Rev. A 72, 042318 (2005).

[21] W. K. Wootters, Phys. Rev. Lett. 80, 2245 (1998).

[22] G. Gour, Phys. Rev. A , 71, 012318 (2005).

[23] It is interesting to note, however, that it is not always straightforward to equate loss of information with mixing 9].

[24] The question remains open for mixed tripartite states.

[25] Another example of 2-way classical communication yielding an advantage over 1-way classical communication is the fact that the 2-way distillable entanglement is greater than the 1-way distillable entanglement for certain mixed states [17].

[26] One can consider imposing restrictions on the nature of the local operations, such as allowing only projective measurements, in order to facilitate the optimization, as has been done in Ref. [7], but we shall not do so here. 